\title{
Development of Langmuir Probes on Divertor Cassettes in JT-60SA
}

\author{
Masakatsu FUKUMOTO, Shinji SAKURAI, Nobuyuki ASAKURA ${ }^{1)}$ and Kiyoshi ITAMI \\ Japan Atomic Energy Agency, Naka, Ibaraki 311-0193, Japan \\ 1) Japan Atomic Energy Agency, Rokkasho, Aomori 039-3212, Japan
}

(Received 10 May 2013 / Accepted 28 August 2013)

\begin{abstract}
Langmuir probes installed in the lower divertor region under high heat flux have been developed and manufactured for JT-60SA. A probe electrode with a head having a rooftop shape is made of a carbon fiber composite and can withstand heat fluxes of up to $10 \mathrm{MW} / \mathrm{m}^{2}$ for $5 \mathrm{~s}$ and $1 \mathrm{MW} / \mathrm{m}^{2}$ for $100 \mathrm{~s}$. This has been achieved by increasing the volume of the probe electrode that is not directly exposed to the plasma. To minimize the reduction of the heat removal performance of the divertor, the Langmuir probes are installed in toroidal gaps with widths of $10 \mathrm{~mm}$ between the divertor cassettes, without embedding them in the divertor tiles. Aluminum oxide coatings have been applied to insulate the probe electrodes from the divertor cassettes and to limit the toroidal thickness to $8 \mathrm{~mm}$. Brazing of the nickel connectors to the probe electrodes has reduced the toroidal thickness of the Langmuir probes. A minimum spatial resolution of $13.5 \mathrm{~mm}$ has been achieved to the Langmuir probes installed on the inner and outer divertor targets.
\end{abstract}

(c) 2013 The Japan Society of Plasma Science and Nuclear Fusion Research

Keywords: Langmuir probe, $\mathrm{Al}_{2} \mathrm{O}_{3}$ coating, brazing, JT-60SA, divertor plasma, plasma equilibrium

DOI: $10.1585 /$ pfr.8.1405153

\section{Introduction}

A Langmuir probe is an essential diagnostic for measuring the electron density $\left(n_{\mathrm{e}}\right)$, electron temperature $\left(T_{\mathrm{e}}\right)$, floating potential $\left(V_{\mathrm{f}}\right)$, and ion saturation current $\left(I_{\mathrm{is}}\right)$ of boundary plasmas. It has been widely used in the scrapeoff layer (SOL) and the divertor regions in many fusion experimental devices to investigate the SOL and divertor plasma physics, such as the plasma transport [1-3] and radiative and detached divertor plasmas [2-4].

JT-60SA has being constructed under the Broader Approach agreement between the Japanese government and European Union [5]. Since high-density and highconfinement plasmas can be sustained for up to $100 \mathrm{~s} \mathrm{[6],}$ the heat and particle loads on the divertor have to be measured over a long duration. In JT-60SA, Langmuir probes are installed on the lower divertor at the start of the operation to investigate the heat and particle load on the divertor. In addition, the Langmuir probes are to be used to verify the plasma equilibrium control upon the commissioning of JT-60SA.

In the current tokamaks, most of the Langmuir probes installed at the divertor are embedded in the divertor tiles, and only the electrode heads protrude from the tile surfaces, protecting the probes from the high heat flux of the divertor plasmas $[7,8]$. This method has the advantage that the Langmuir probes can be replaced in the vacuum vessel after the divertor tiles are removed from the plasma side.

The divertor cassettes are equipped with water-cooled

author'se-mail: fukumoto.masakatsu@jaea.go.jp copper alloy heat sinks with carbon fiber composite (CFC) armor tiles. Since a part of the divertor tiles is bolted from the back side of the heat sinks, the tiles cannot be removed in the vacuum vessel from the plasma side. In addition, inspection and repair of the divertor are performed outside the vacuum vessel after the divertor cassettes are taken out using the remote handling systems [9]. Therefore, it is difficult to replace the Langmuir probes in the vacuum vessel. For JT-60SA, embedding the Langmuir probes in the divertor tiles only leads to reduction of the heat removal performance of the divertor.

In this study, the Langmuir probes that can be installed on the lower divertor in JT-60SA without needing to embed them in the divertor tiles have been developed and manufactured. In this paper, the design requirements are described in Sec. 2. The manufactured Langmuir probes are described in Sec. 3, and their installation and cabling to the divertor cassettes are also explained. Power supplies and data acquisition are mentioned briefly in Sec. 3. Section 4 describes the evaluation of the ion saturation current and the measurable range of the plasma parameters. A thermal analysis of the probe electrode is discussed in Sec. 5. Section 6 describes the adhesion of the brazing applied to join the connectors to the probe electrodes in order to connect the electrical cables to the electrodes. The conduction between the probe electrodes and connectors is also described. The evaluation of the insulating resistance of an aluminum oxide $\left(\mathrm{Al}_{2} \mathrm{O}_{3}\right)$ coating, which was applied to insulate the probe electrodes electrically from the divertor cassettes, is described in Sec. 7. Section 8 gives a summary 
on the development of Langmuir probes in this work.

\section{Design Requirements}

To measure the distributions of the plasma parameters, for example, the electron density, temperature, particle flux, and floating potential, the Langmuir probes have to be installed at intervals within the scale lengths of these parameters. The power flux density to the target surface, $q$, has relation with these parameters as follows [10]:

$$
q \propto T \Gamma \propto T^{3 / 2} n,
$$

where $T$ is the temperature, $\Gamma$ is the particle flux density, and $n$ is the density. The floating potential is proportional to the electron temperature [11]. Therefore, the power decay length is estimated to be shorter than the scale lengths of the plasma parameters. The spatial resolution of the Langmuir probes on the divertor targets is determined to be higher than the power decay length at the outer midplane in consideration of the magnetic flux expansion at the outer divertor target. The power decay length at the outer mid-plane, $\lambda_{\mathrm{q}}$, is evaluated using the following equation [12]:

$$
\begin{aligned}
\lambda_{\mathrm{q}}= & 1.63 \times\left(R_{0} / 2.9\right)^{0.7 \pm 0.6} \\
& \times B^{-0.57 \pm 0.31} q_{\mathrm{cyl}}^{1.0 \pm 0.31} P_{\text {sol }}^{0.23 \pm 0.09} Z^{0.3 \pm 0.1}(\mathrm{~mm}),
\end{aligned}
$$

where $R_{0}$ is the major radius, $B$ is the toroidal magnetic field, $P_{\text {sol }}$ is the power crossing the separatrix, and $Z$ is the charge number of the fuel ion. The edge safety factor, $q_{\text {cyl }}$, is expressed as follows:

$$
q_{\mathrm{cyl}}=\frac{2 \pi \varepsilon \kappa a B}{\mu_{0} I_{\mathrm{p}}},
$$

where $\varepsilon=a / R_{0}$ is the inverse tokamak aspect ratio ( $a$ is the minor radius), $\kappa$ is the elongation, $\mu_{0}$ is the magnetic permeability, and $I_{\mathrm{p}}$ is the plasma current. The reference parameters of JT-60SA for a full current inductive scenario with a single null divertor are as follows:

$$
\begin{aligned}
& a \sim 1.18 \mathrm{~m}, R_{0} \sim 2.96 \mathrm{~m}, \varepsilon \sim 0.4, \\
& \kappa \sim 1.72, B \sim 2.25 \mathrm{~T}, \text { and } I_{\mathrm{p}} \sim 5.5 \mathrm{MA} .
\end{aligned}
$$

The power crossing the separatrix is assumed to be $85 \%$ of the heating power $\left(P_{\text {heat }}=41 \mathrm{MW}\right)$ based on the collected data for $P_{\text {sol }}$, with reference to $P_{\text {heat }}[12]$. By inserting the above parameters into Eqs. (2) and (3), the power decay length at the JT-60SA outer target is evaluated as $\lambda_{\mathrm{q}}=13.3-57.4 \mathrm{~mm}$ in consideration of the magnetic flux expansion of $\sim 7$. Hence, the spatial resolution of the Langmuir probes on the divertor targets requires to be $\sim 13 \mathrm{~mm}$.

The maximum heat fluxes to the inner and outer divertor targets are 1 and $10 \mathrm{MW} / \mathrm{m}^{2}$ for 100 and $5 \mathrm{~s}$, respectively. These values depend on the heat removal capability of the divertor targets. Hence, the Langmuir probes installed on the divertor targets are required to withstand the high heat load and measure the plasma parameters correctly. The heat flux to the other regions including the inner

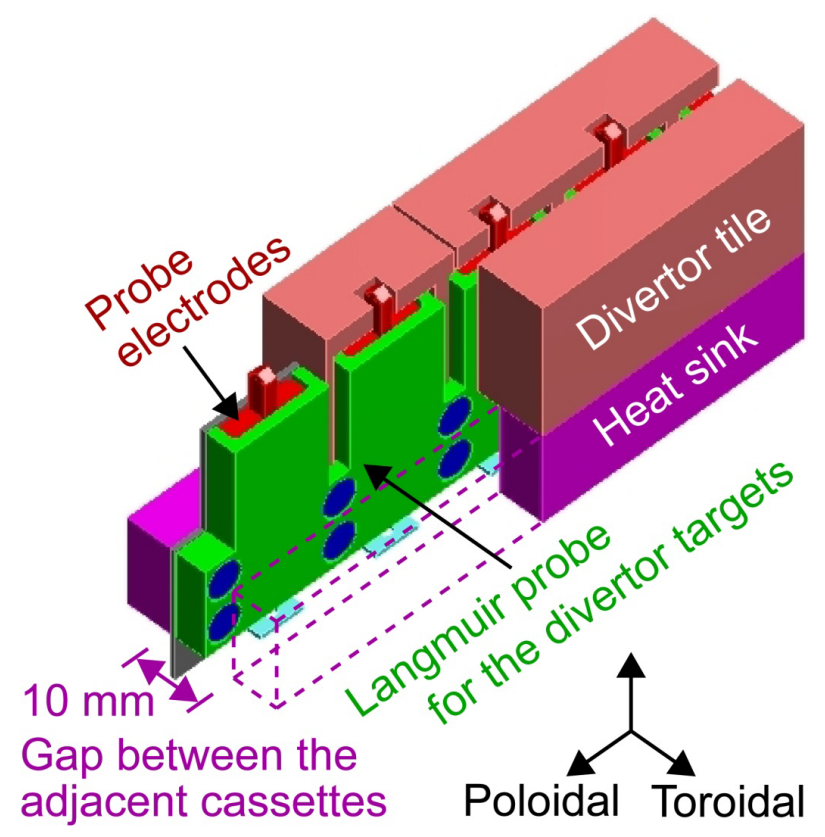

Fig. 1 Langmuir probes installed on the lateral side of the watercooled copper alloy heat sink. The heat sink equipped with the Langmuir probe is installed on the toroidal edge of the divertor cassette.

and outer baffles, the private dome, and the cover for the pipe connection are $2 \mathrm{MW} / \mathrm{m}^{2}$ or less for a $100 \mathrm{~s}$ duration.

Since the electrode heads protrude from the divertor tiles to collect plasma particles, the electrode head is eroded, and this erosion results in an error in the measured signal. To reduce this error, the probe electrodes have to be replaced periodically. In this development, the Langmuir probes are designed to be installed with bolts on the lateral side of the water-cooled heat sinks, which are installed on the toroidal edge of the divertor cassettes, as shown in Fig. 1. This provides the minimum reduction of the heat removal performance of the divertor. In addition, the Langmuir probes can be replaced easily after the divertor cassettes are removed from the vacuum vessel.

As shown in Fig. 1, the toroidal gap width between the divertor cassettes is $\sim 10 \mathrm{~mm}$. Since a $2-\mathrm{mm}$ gap width is required to install the divertor cassettes in the vacuum vessel with the remote handling systems, the toroidal thickness of the Langmuir probes has to be limited to $8 \mathrm{~mm}$ or less.

\section{Structure and Installation of the Langmuir Probes}

\subsection{Structure}

Four sets of 44-channel Langmuir probes have been manufactured for the JT-60SA lower divertor. As an example, Fig. 2 (a) shows Langmuir probes installed on the lower portion of the divertor targets. The Langmuir probes consist of probe electrodes with brazed connectors, an 

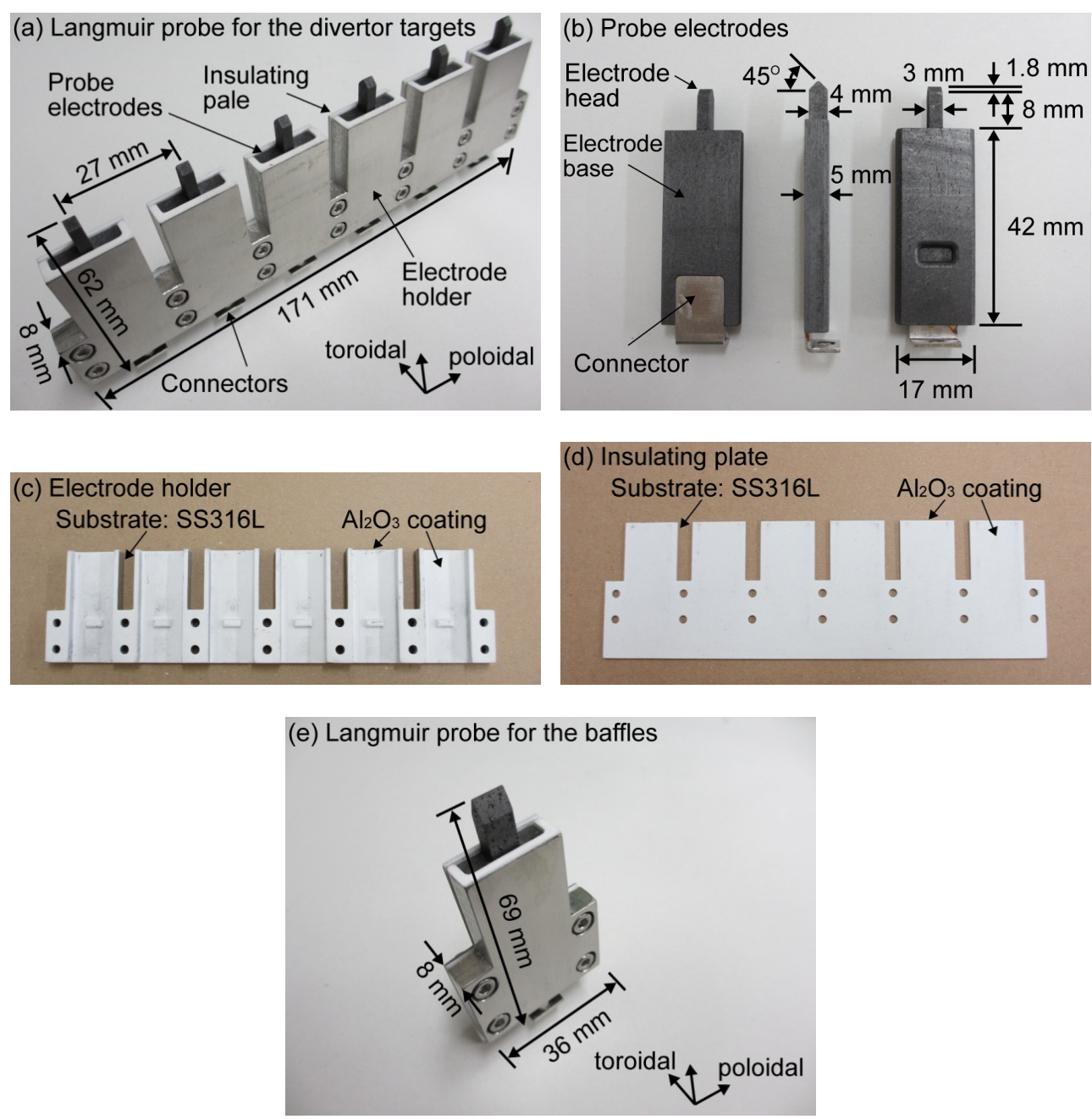

Fig. 2 (a) Langmuir probes installed in the lower portion of the divertor targets. The toroidal and poloidal directions are shown in the lower right. (b) An electrode holder and (c) an insulating plate. $\mathrm{An}^{\mathrm{Al}_{2}} \mathrm{O}_{3}$ coating is applied to the inner surface and top side of the electrode holder and insulating plate, both of which are made of SS316L. (d) Probe electrodes taken from the insulating plate side (left), the poloidal direction (center), and the electrode holder side (right). A connector for a mineral insulation (MI) cable is brazed to the lower end of the probe electrode. (e) A Langmuir probe for the inner and outer baffles.

electrode holder, and an insulating plate. The toroidal width is limited to $8 \mathrm{~mm}$. The poloidal length and height are 171 and $62 \mathrm{~mm}$, respectively. The distance between the probe heads is $27 \mathrm{~mm}$.

Figure 2(b) shows the probe electrodes with the brazed connectors for the Langmuir probes installed on the divertor targets. The electrode is made of CFC. Since a high heat flux of up to $10 \mathrm{MW} / \mathrm{m}^{2}$ is applied to the divertor targets, CX-2002U (produced by Toyo Tanso), which has a high thermal conductivity $(\sim 310 \mathrm{~W} / \mathrm{m} \mathrm{K})$, is selected as the probe electrode material. For a lower heat flux $\left(2 \mathrm{MW} / \mathrm{m}^{2}\right)$ region, including the inner and outer baffles, the private dome, and the cover for the pipe connection, PCC-2S (produced by Hitachi Chemical Co., Ltd.), which has a lower thermal conductivity $(\sim 250 \mathrm{~W} / \mathrm{m} \mathrm{K})$, is used since the PCC-2S is more economical than the CX-2002U. The electrode head is given a "rooftop" shape [8]. This shape has the advantage that the incident heat and parti- cle fluxes are evenly distributed compared with the domeshaped electrode head previously used in JT-60U. The dimensions of the electrode head of the Langmuir probes are $4 \mathrm{~mm}$ in the toroidal direction and $3 \mathrm{~mm}$ in the poloidal direction. The angle and height of the rooftop are $45^{\circ}$ and $\sim 1.8 \mathrm{~mm}$, respectively, with respect to the target tile surface. To reduce the temperature increase of the rooftop during plasma discharges, the electrode base, which is not irradiated by plasmas directly, is expanded poloidally to $17 \mathrm{~mm}$.

The connector for a mineral insulation (MI) cable is brazed to the lower end of the probe electrode. Since the temperature at the lower end of the probe electrode reaches $\sim 350^{\circ} \mathrm{C}$, which is the softening temperature of pure copper, pure nickel is selected as the connector material. This brazing also reduces the toroidal thickness of the Langmuir probes.

Figures 2 (c) and (d) show the electrode holder and in- 
sulating plate, respectively. Since the toroidal gap between the divertor cassettes is $10 \mathrm{~mm}$, the electrode holder and insulating plate must be thin and retain mechanical strength at temperatures above $200^{\circ} \mathrm{C}$ (the baking temperature of the vacuum vessel). In addition, the probe electrodes have to be insulated electrically from the divertor cassettes. This has been achieved by using an aluminum oxide $\left(\mathrm{Al}_{2} \mathrm{O}_{3}\right)$ coating with a nickel and 5\%-aluminum inter-layer on the inner surface of the electrode holder and that of the insulating plate, which are both made of stainless steel, SS 316L. The thickness of the $\mathrm{Al}_{2} \mathrm{O}_{3}$ coating is $200-400 \mu \mathrm{m}$. The nickel and 5\%-aluminum inter-layer, which has a thickness of $30-50 \mu \mathrm{m}$, is applied to prevent crack formation in the $\mathrm{Al}_{2} \mathrm{O}_{3}$ coating. It is expected that the carbon that is eroded mainly from the first wall and divertor tiles by plasma discharges is deposited on the top of the electrode holder and insulating plate. The deposited carbon connects the probe electrodes to the heat sink electrically through the electrode holder and insulating plate. Hence, the $\mathrm{Al}_{2} \mathrm{O}_{3}$ with the nickel and 5\%-aluminum inter-layer is also coated on the top side of the electrode holder and insulating plate to prevent the electrical connection.

The Langmuir probes for the divertor targets are in an array configuration since fine spatial resolution $(\sim 13 \mathrm{~mm})$ is required. Since the other regions are accommodated to a coarse spatial resolution compared with that of the divertor targets, separate individual Langmuir probes are installed, as shown in Fig. 2 (e). Note that the poloidal widths of the probe holder and the insulating plate for the private dome and the cover for the pipe connection are increased compared with those for the baffles to avoid interference of the fixing bolts with the flow channel of the coolant water.

\subsection{Installation to the divertor cassette}

The torus is composed of 18 toroidal sections [13]. The toroidal width of the divertor cassette is $10^{\circ}$, and two divertor cassettes are installed in one toroidal section. Figures 3 (a) and (b) show the Langmuir probes installed on the toroidal edge of one divertor cassette. The directions of the plasma current and toroidal field are normally clockwise when viewed from the top of the torus. In this case, the directions of the SOL plasma flow at the high-field side and low-field side are clockwise and counter-clockwise, respectively. In the case that the Langmuir probes are installed on the toroidal edge of the divertor cassette that corresponds to the upstream side of the SOL plasma flow, ions and electrons impinge on the lateral side of the electrode head in addition to the rooftop due to the gap between the divertor cassettes $(\sim 10 \mathrm{~mm})$. To reduce the number of ions and electrons impinging on the lateral side of the electrode head, the Langmuir probes are installed on the downstream side of the SOL plasma flow, and the divertor tiles of the upstream side are cut around the electrode head, as shown in Fig. 3 (c).

Figure 4 shows the spatial resolution of the Langmuir
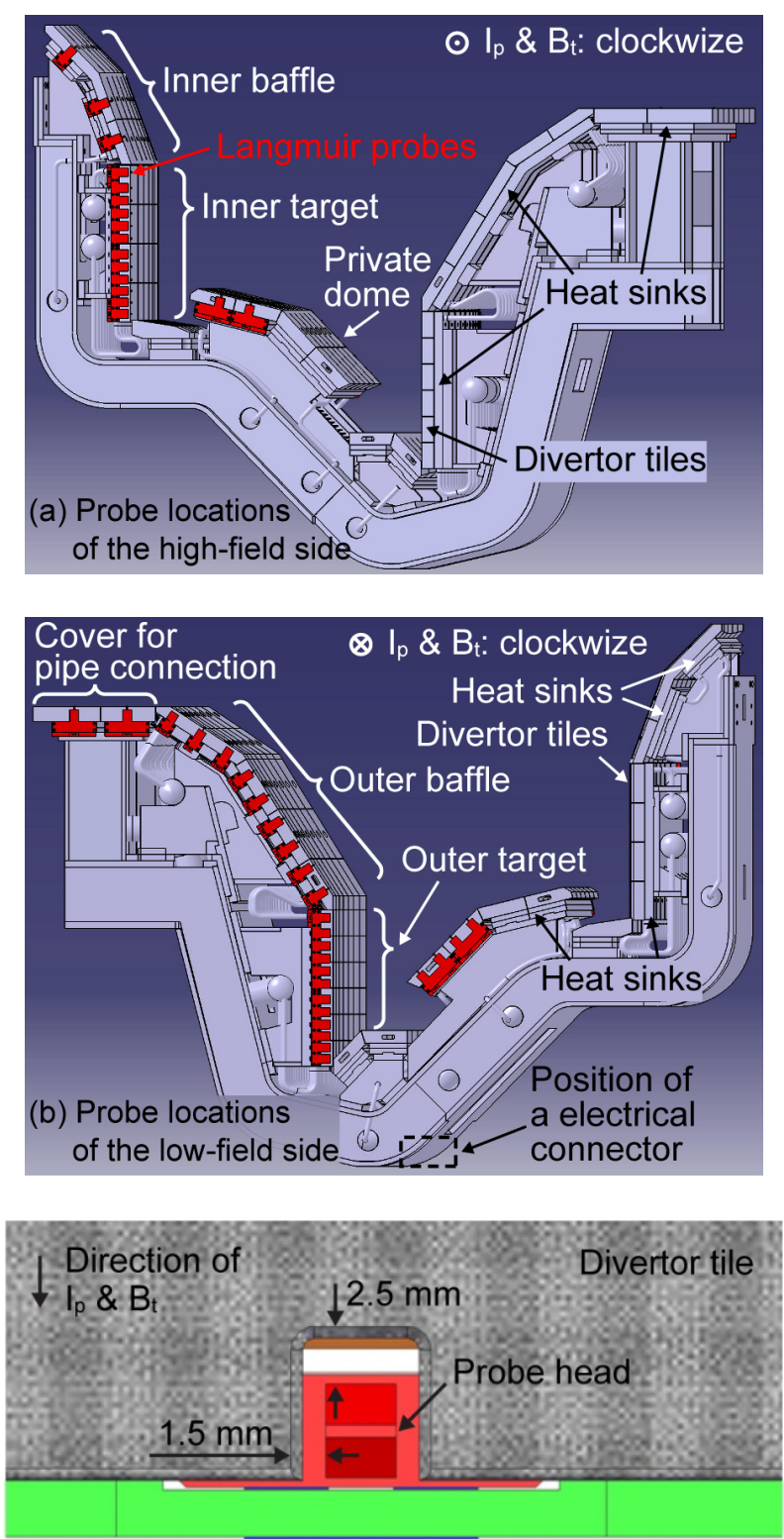

(c) Expanded top view around the probe head

Fig. 3 (a), (b) The Langmuir probes installed on the toroidal edge of the divertor cassettes, which correspond to the upstream side of the SOL plasma flow. The rectangle drawn with a dashed line in the bottom portion of the divertor cassette indicates the position at which the electrical connector is installed. (c) Magnified top view around the electrode head of the target Langmuir probe.

probes installed on the inner and outer divertor targets. The Langmuir probes are installed at the 4 toroidal positions in a total of 18 toroidal sections, including Sections P-3, 4, 9, and 15. To increase the spatial resolution, the Langmuir probes in Sections P-3 and 9 are installed $13.5 \mathrm{~mm}$ above those in Sections P-4 and 15. This improves the spatial resolution to $13.5 \mathrm{~mm}$ at the divertor targets in the case that the Langmuir probes in Section P-3 or 9 and Section P-4 or 15 are used. Although, this spatial resolution is $\sim 0.5 \mathrm{~mm}$ larger than the requirement determined in the 
(a) Inner divertor targets

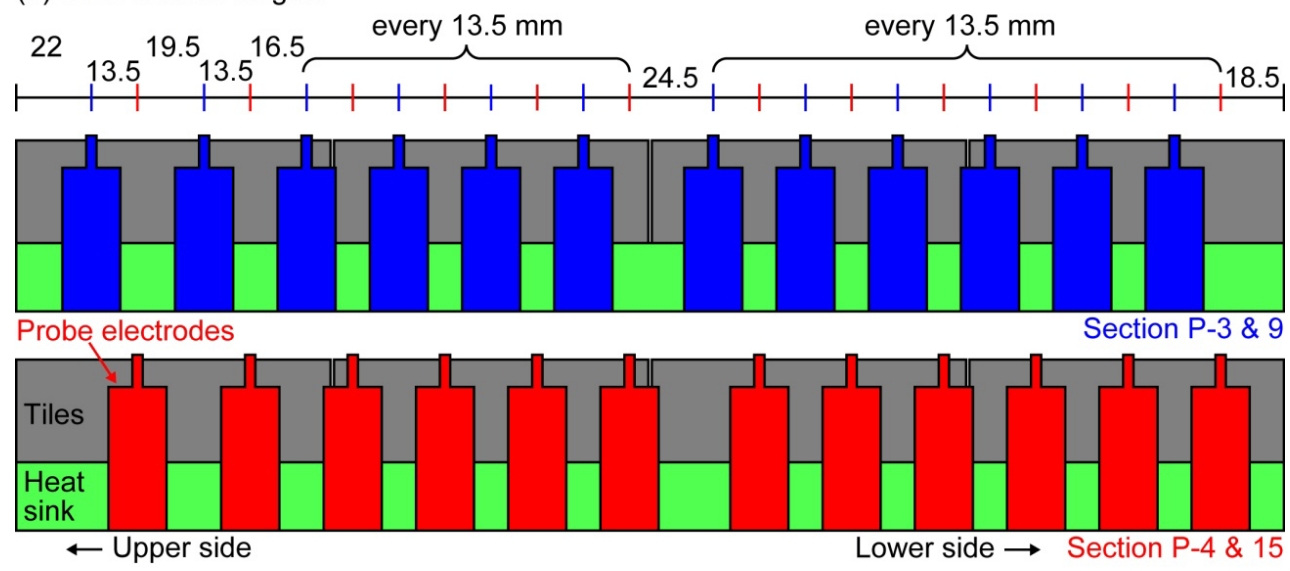

(b) Outer divertor targets

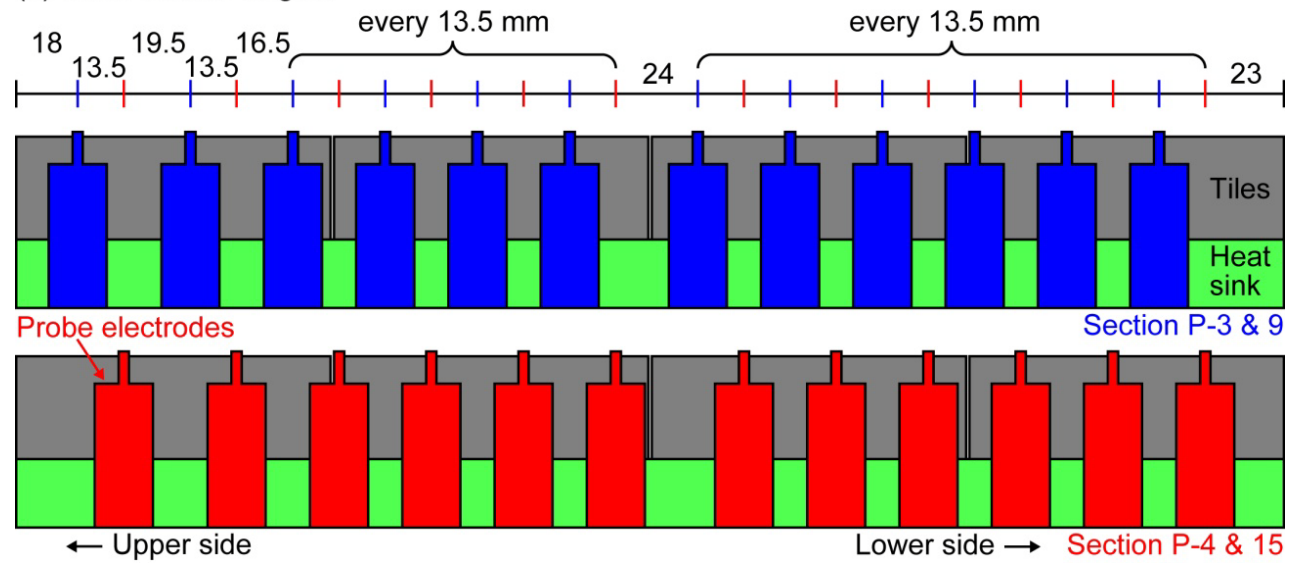

Fig. 4 Spatial resolution of the Langmuir probes installed on (a) the inner and (b) outer divertor targets.

previous section $(\sim 13 \mathrm{~mm})$, the poloidal distribution of the plasma parameters should be measured for most plasma discharges.

The spatial resolution of the outer baffle is determined based on the radial distribution of the electron density at the outer mid-plane calculated by the SONIC code [14] for the full current inductive scenario [15]. The radial distribution is extrapolated to the outer baffle region in consideration of the magnetic flux expansion. The Langmuir probes are installed on the outer baffle at $65-80 \mathrm{~mm}$ intervals, which is shorter than the decay length of the electron density of $\sim 100 \mathrm{~mm}$. The spatial resolution on the private dome is similar to that on the outer baffle. The interval on the cover for the pipe connection is about twice as large as that on the outer baffle $(\sim 125 \mathrm{~mm})$. Only three Langmuir probes are installed on the inner baffle since the bolt holes interfere with the flow channel of the coolant water. However, the intervals of $90-160 \mathrm{~mm}$ are shorter than the decay length of the electron density $(\sim 200 \mathrm{~mm})$.

The toroidal positions of the Langmuir probes are chosen so that the divertor study is conducted in combination with other diagnostics. Since a divertor Thomson scattering system is installed in the Section P-2 divertor region, the Langmuir probes installed in Section P-3 provide a comparison of the plasma parameters. The surface temperature of the divertor region, especially the outer divertor target of Section P-4, is measured by a tangentially viewing divertor infrared (IR) TV camera installed on an equatorial diagnostic port positioned in Section P-18. Hence, the heat and particle loads and their distributions on the outer divertor target are compared at the same position on the divertor target. A material introduction system, which can expose materials to the divertor plasmas for the study of plasma surface interactions, is installed on the Section P-10 lower divertor. The Langmuir probes installed in Section P-9 can measure the plasma parameters at the neighbor toroidal position during sample exposure. Section P-15 corresponds to the toroidal position which is trisected by Sections P-3 and 9.

\subsection{Cabling on the divertor cassette}

A mineral insulation (MI) cable with an oxygen-free copper conductor inside a stainless steel (SS316L) sheath that is insulated by silicon dioxide $\left(\mathrm{SiO}_{2}\right)$ is used as an electrical cable in the vacuum vessel. The electrical signals are transmitted outside of the vacuum vessel using a feedthrough mounted on a diagnostic port. To remove the divertor cassettes from the vacuum vessel without cutting 
the MI cables, an electrical connector is installed on the bottom portion of the divertor cassette and the vacuum vessel, as shown in Fig. 3 (b). The MI cables join the connectors of the Langmuir probes and the electrical connector and are fixed along the frame of the divertor cassette. The thickest MI cable that can be fixed in the narrow space and complicated structure of the divertor cassette is selected for the Langmuir probe installed on the divertor targets. The diameters of the cable and the conductor are $\sim 2.3$ and $\sim 0.82 \mathrm{~mm}$, respectively, and the allowable current is $\sim 5.7$ A. For the Langmuir probes installed at the other positions, a thinner MI cable is selected since a lower current is expected. The diameters of the cable and the conductor are $\sim 1.6$ and $\sim 0.54 \mathrm{~mm}$, respectively, and the allowable current is $\sim 2.5 \mathrm{~A}$. Similar MI cables are used to join the divertor connectors and the feedthroughs on the diagnostic port of the vacuum vessel.

\subsection{Power supplies and data acquisition}

The power supplies used in the JT-60U Langmuir probe systems are reused for the JT-60SA. The maximum current is $\sim 4 \mathrm{~A}$, including for a protection circuit such as a fuse. The voltage of a triangular wave applied to the probe electrodes varies from -160 to $40 \mathrm{~V}$ with a frequency of up to $1 \mathrm{kHz}$. We aim to acquire data over a duration of more than $100 \mathrm{~s}$, sampled such that there are about 400 points for each period of the applied voltage wave. This is to be achieved by upgrading the data acquisition systems.

\section{Evaluation of the Ion Saturation Current}

The ion saturation current was evaluated for the Langmuir probe installed on the divertor targets. Figure 5 shows the electron density dependence of the ion saturation cur-

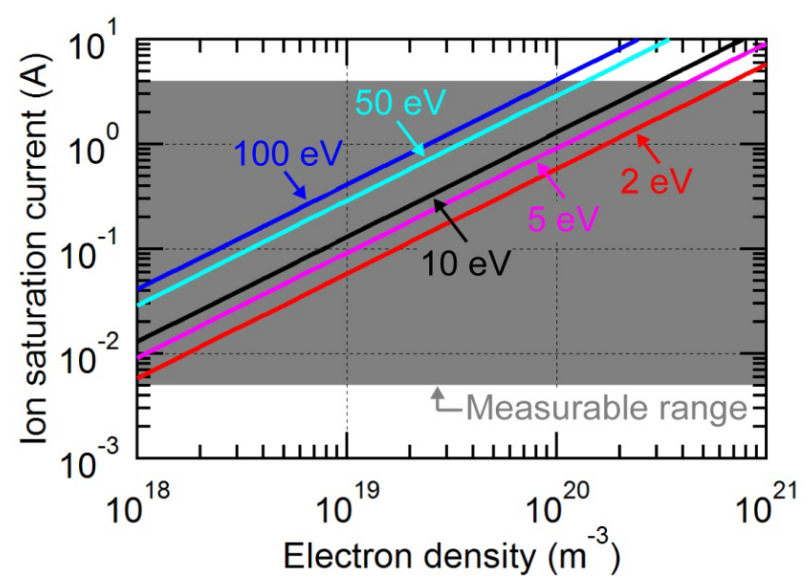

Fig. 5 Electron density dependence of the ion saturation current for five different values of the electron temperature. The gray color indicates the measurable range of the Langmuir probes installed on the targets. rent for five electron temperature values. The electron density ranges from $10^{18}$ to $10^{21} \mathrm{~m}^{-3}$. The electron temperature is assumed to range from 2 to $100 \mathrm{eV}$. The maximum current of the probe electrode is limited by the power supplies $(\sim 4 \mathrm{~A})$. The minimum current of $\sim 5 \mathrm{~mA}$ is required to perform curve fitting to the current-voltage characteristics since the same current is necessary for the JT-60U Langmuir probes with a similar surface area $\left(\sim 6 \mathrm{~mm}^{2}\right)$ as the electrode heads.

In the case that the electron density is less than $10^{20} \mathrm{~m}^{-3}$, the ion saturation current falls in the measurable range. When the electron density is higher than $10^{20} \mathrm{~m}^{-3}$, the maximum electron density increases with decreasing electron temperature: the electron density increases to $\sim 8 \times 10^{20} \mathrm{~m}^{-3}$ for an electron temperature of $2 \mathrm{eV}$.

The poloidal distribution of the electron density and temperature on the divertor targets were calculated for the peak heat load of $10 \mathrm{MW} / \mathrm{m}^{2}$ [15]. Under the detached divertor plasma condition, the maximum electron density and temperature are $\sim 1.5 \times 10^{21} \mathrm{~m}^{-3}$ and $\sim 2 \mathrm{eV}$, respectively. In this case, the ion saturation current exceeds the measurable range of the Langmuir probe for the divertor targets. Note that, although rollover and decrease of the incident ion flux to the divertor target have been observed [16-18], these phenomena are not reproduced using the SONIC code [19]. Hence, since the ion saturation current evaluated based on the plasma parameter obtained by SONIC code is overestimated, the ion saturation current under the detached divertor plasma condition would fall in the measurable range. Under the attached divertor plasma condition, electron density and temperature change from $\sim 10^{18}$ to $\sim 9 \times 10^{19} \mathrm{~m}^{-3}$ and $10-40 \mathrm{eV}$, respectively. In this case, the ion saturation current falls in the measurable range. Therefore, the Langmuir probes installed on the divertor targets can measure the plasma parameters expected on the divertor targets in JT-60SA.

The electron density and temperature on the inner and outer baffles, the private dome, and the cover for the pipe connection are expected to be low compared with those on the divertor targets. The surface area of the rooftop is expanded to obtain the detectable ion saturation current, which is evaluated based on the radial distribution of the electron density and temperature at the inner and outer mid-plane calculated by the SONIC code for the peak heat load of $10 \mathrm{MW} / \mathrm{m}^{2}$ in consideration of the magnetic flux expansion.

The electron density and temperature on the inner and outer baffles change from $\sim 5 \times 10^{17}$ to $\sim 9 \times 10^{18} \mathrm{~m}^{-3}$ and less than $10 \mathrm{eV}$, respectively. To detect ion saturation currents over $5 \mathrm{~mA}$, the dimensions of the electrode head are increased to $6 \mathrm{~mm}$ in the toroidal direction and $4 \mathrm{~mm}$ on the plasma side. The effective surface area of the rooftop is $\sim 22.5 \mathrm{~mm}^{2}$ in the case that the pitch angle of the incident ions is assumed to be $10^{\circ}$. The dimensions of the electrode head for the private dome are the same as those for the baffles. 
Table 1 Properties of the CFC used in the thermal analysis.

\begin{tabular}{ll}
\hline Item & Value \\
\hline Density & $1.7 \mathrm{~g} / \mathrm{cm}^{3}$ \\
Specific heat & $710 \mathrm{~J} / \mathrm{kg} / \mathrm{deg}$. \\
Thermal conductivity & $310\left(25^{\circ} \mathrm{C}\right)-120 \mathrm{~W} / \mathrm{m} \mathrm{K}\left(1000^{\circ} \mathrm{C}\right)$ \\
Emissivity & 0.7 \\
\hline
\end{tabular}

At the cover for the pipe connection, the electron density and temperature are on the order of $10^{17} \mathrm{~m}^{-3}$ and less than $5 \mathrm{eV}$, respectively. The dimensions of the electrode head are increased to $6 \mathrm{~mm}$ in the toroidal direction and $5 \mathrm{~mm}$ on the plasma side. The effective surface area of the rooftop is increased to $\sim 31.4 \mathrm{~mm}^{2}$ for a pitch angle of $10^{\circ}$. Since the ion saturation current is evaluated to be on the order of $0.1 \mathrm{~mA}$, an amplifier and a noise filter would be required to perform the curve fitting to the current-voltage characteristics.

\section{Thermal Analysis of the Probe Electrode}

Thermal analysis of the probe electrode for the Langmuir probes installed on the divertor targets was performed. The divertor targets designed with bolted CFC armor tiles and a water-cooled heat sink can handle heat flux values of 1 and $10 \mathrm{MW} / \mathrm{m}^{2}$ for 100 and $5 \mathrm{~s}$, respectively. For each case, the time evolution of the temperature of the rooftop and the brazing position of the connector was calculated with the finite element method (FEM). For comparison, the same analysis was performed for a square pillar electrode with the same length. The initial temperature of the probe electrode is set to be $200^{\circ} \mathrm{C}$, which corresponds to the baking temperature of the vacuum vessel. The heat conduction of the probe electrode to the surroundings is assumed to be adiabatic. It is assumed that the heat flux impinges on half of the rooftop from a direction parallel to the tile surface. The properties of the CFC used in this analysis are listed in Table 1.

Figure 6 shows the time evolution of the temperature of the rooftop and the brazing position of the connector for the heat flux of $1 \mathrm{MW} / \mathrm{m}^{2}$ for $100 \mathrm{~s}$. In the case of the square pillar electrode, the temperatures of the rooftop and the brazing position increase to $\sim 620$ and $\sim 500^{\circ} \mathrm{C}$, respectively. In the case that the electrode base is expanded poloidally, the rate of increase of the rooftop temperature begins to decrease at $1 \mathrm{~s}$ compared with the case of the square pillar electrode. This is because the heat flux diffuses to the boundary between the electrode head and the electrode base and begins to spread poloidally in the electrode base. The expansion of the electrode base limits
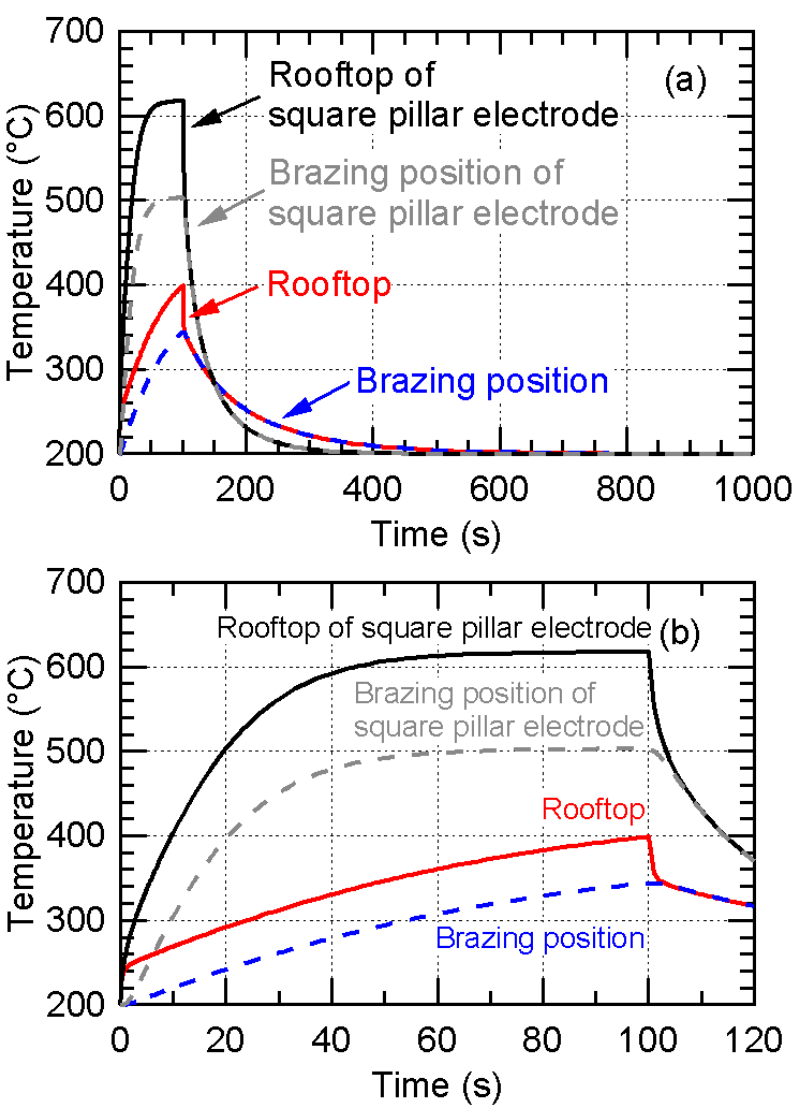

Fig. 6 Time evolution of the temperature of the rooftop and brazing position of the connector for a heat flux of $1 \mathrm{MW} / \mathrm{m}^{2}$ for $100 \mathrm{~s}$. The top panel (a) shows data for a $1000 \mathrm{~s}$ duration. The bottom panel (b) shows a magnified view, limited to data for a $120 \mathrm{~s}$ duration.

the temperatures of the rooftop and the brazing position to $\sim 400$ and $\sim 340^{\circ} \mathrm{C}$, respectively.

Figure 7 shows the time evolution of the temperatures of the rooftop and the brazing position of the connector for a heat flux of $10 \mathrm{MW} / \mathrm{m}^{2}$ for $5 \mathrm{~s}$. Although the calculation was terminated at $3.4 \mathrm{~s}$ for the square pillar electrode, the rooftop temperature increased to $\sim 1900^{\circ} \mathrm{C}$ or more, at which temperatures the thermionic electron emission is enhanced. In the case that the electrode base is expanded poloidally, the rooftop temperature is limited to $\sim 950^{\circ} \mathrm{C}$ 
which is sufficiently low to avoid the thermionic electron emission. The temperature of the brazing position is also decreased from $\sim 400^{\circ} \mathrm{C}$ or more to $\sim 300^{\circ} \mathrm{C}$.

To prevent the temperature increase caused by repetition of plasma discharges, the electrode temperature needs to be decreased to the initial temperature $\left(200^{\circ} \mathrm{C}\right)$ during the intervals between plasma discharges. The nominal repetition time of the discharge is determined by the cooling time of the power supplies for the neutral beam injection (NBI) system. The duty cycle of the NBI is expected to be $1 / 30$ during the flat-top phase of the NBI. In the case of a 100 -s flat top (heat flux of $1 \mathrm{MW} / \mathrm{m}^{2}$ ), the probe electrode temperature decreases to $200^{\circ} \mathrm{C}$ until the next plasma discharge, as shown in Fig. 6 (a). In the case of a 5-s flat top (heat flux of $10 \mathrm{MW} / \mathrm{m}^{2}$ ), the probe electrode temperature at $150 \mathrm{~s}$ decreases only to $\sim 240^{\circ} \mathrm{C}$, as shown in Fig. 7 (a), which is higher than the initial temperature. However, since the intervals between the plasma discharges will be prolonged to more than $10 \mathrm{~min}$ by data transfer based on those in JT-60U, the temperature of the probe electrode should decrease to $200^{\circ} \mathrm{C}$ until the next plasma discharge.
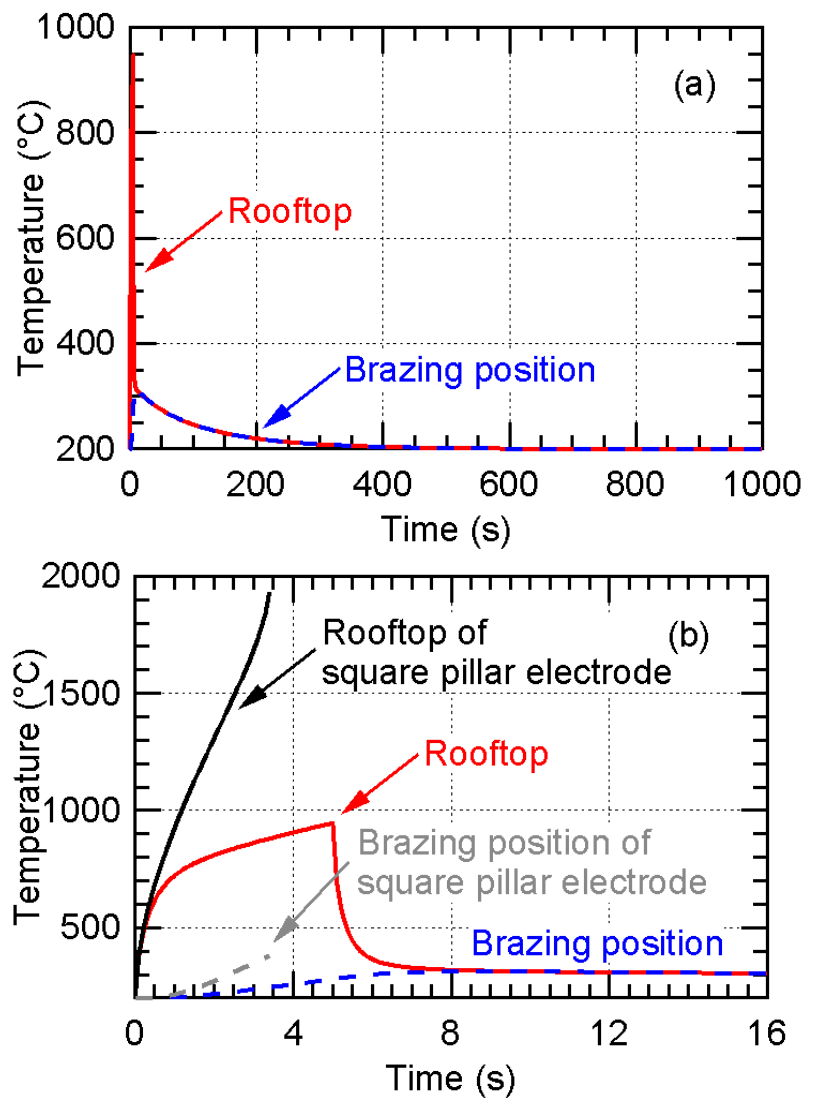

Fig. 7 Time evolution of the temperature of the rooftop and brazing position of the connector for a heat flux of $10 \mathrm{MW} / \mathrm{m}^{2}$ for $5 \mathrm{~s}$. The top panel (a) shows data for a $1000 \mathrm{~s}$ duration. The bottom panel (b) shows a magnified view, limited to data for a $16 \mathrm{~s}$ duration.

\section{Brazing of Nickel to the CFC and Conduction of the Blazed Samples}

Although it is necessary to braze the connectors to the probe electrodes to reduce the toroidal thickness of the Langmuir probes, there is no known practical method for brazing nickel to the CFCs (CX-2002U and PCC-2S). Hence, adhesion between nickel and the CFCs was investigated by cross-sectional observation of the brazed interface. In addition, it was confirmed that the conduction between the connectors and probe electrodes measured by an ohmmeter is $0.2 \Omega$ or less.

The nickel was brazed to the CFCs using nickel-based filler metal composed of $\sim 12 \%$ phosphorus. This brazing filler metal is widely used to braze metal to carbon. The nickel brazed to the CFCs was removed easily at the interface between the brazing filler metal and CFCs. This was because the brazing filler metal did not spread uniformly between the nickel and CFCs due to low reactivity and wettability of the brazed filler metal with respect to the CFC surface.

To activate the CFC surface and distribute brazing filler metal uniformly, silver-based brazing filler metal composed of $28 \%$ copper and $2 \%$ titanium was selected. Since this brazing filler metal contains the active metal, i.e., titanium, an increase of the reactivity and wettability to the CFC surface is expected. Figure 8 shows a cross section of the brazed interface between nickel and the CFC (PCC-2S). The brazing filler metal is distributed uniformly between the nickel and CFC. It is observed that the interfaces between the nickel and blazing filler metal and between the brazing filler metal and the CFC are joined without any pores, cracks, or interspaces. In addition, the brazing filler metal fills the pores and dimples of the CFC surface. The cross-sectional observation of the brazed interface indicates that the nickel and the CFC are joined solidly.

Since the coefficients of thermal expansion (CTE) of nickel, the brazing filler metal, and CFCs are different, thermal stresses are produced near the interface by tem-

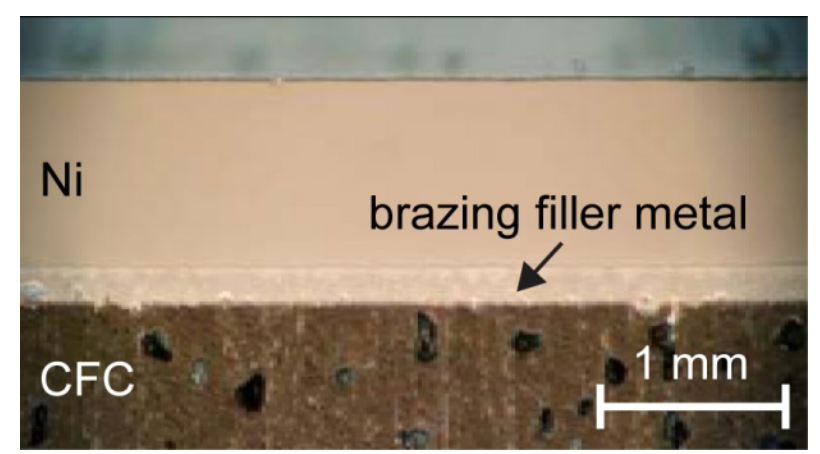

Fig. 8 Cross section of the brazed interface between the nickel and CFC (PCC-2S). 
perature changes. In this case, there is a possibility that the connector will be removed from the brazed interface by the heat cycles from the plasma. To investigate the soundness of the blazing interface and the change of the conduction between the nickel and CFCs, heat-cycle tests of the nickel brazed CFCs were performed.

Three samples using the CFCs of each of CX-2002U and PCC-2S, respectively, were manufactured. The sample size is similar to the probe electrode (Fig. 2 (b)) without the electrode head. The heat-cycle test was performed in vacuum atmosphere $(\sim 0.1 \mathrm{~Pa})$ to up to 300 cycles. The samples were heated from the CFC side (bottom side in Fig. 8). The temperature of the nickel was measured by a thermocouple, which was placed in contact with the nickel surface and fixed with a graphitic adhesive. Since higher temperature leads to greater stress at the interfaces, a temperature increase for the heat flux of $1 \mathrm{MW} / \mathrm{m}^{2}$ for $100 \mathrm{~s}$ was assumed. The nickel temperature was increased from 200 to $400^{\circ} \mathrm{C}$ with a similar rate of increase as that predicted by the FEM analysis (Fig. 6(b)). Visual observation revealed that the nickel is not removed from the CFCs. The conduction measured before and after the heat-cycle test with an ohmmeter showed that no clear degradation between the nickel and the CFCs was observed. Therefore, the brazing of the nickel to the CFCs was used to join the connectors to the probe electrodes.

\section{Insulating Resistance of the $\mathrm{Al}_{2} \mathrm{O}_{3}$ Coating on the SS316L}

The probe electrode is electrically insulated from the heat sink by the $\mathrm{Al}_{2} \mathrm{O}_{3}$ coating on the inner surface of the probe holder and the insulating plate. Although the nickel and 5\%-aluminum inter-layer is coated, there is a possibility that crack and peel-off are formed on the $\mathrm{Al}_{2} \mathrm{O}_{3}$ coating by temperature changes due to the CTE mismatch between the $\mathrm{Al}_{2} \mathrm{O}_{3}$ and the SS316L. These cause deterioration of the insulating resistance of the $\mathrm{Al}_{2} \mathrm{O}_{3}$ coating. Therefore, the insulating resistance of the $\mathrm{Al}_{2} \mathrm{O}_{3}$ coating on the SS316L was measured before and after the heatcycle test, and the insulating resistance was confirmed to be more than $80 \mathrm{M} \Omega$.

Figure 9 shows the $\mathrm{Al}_{2} \mathrm{O}_{3}$-coated $\mathrm{SS} 316 \mathrm{~L}$ sample used for measurement of the insulating resistance before and after the heat-cycle tests. Three samples, the dimensions of which are similar to those of the electrode holder (Fig. 2(c)), were manufactured to measure the insulating resistance of the coating since the electrode holder is difficult to coat compared with the insulating plate. The nickel and 5\%-aluminum inter-layer with a thickness of 30 - $50 \mu \mathrm{m}$ was coated on the SS316L substrate by plasma splaying. An $\mathrm{Al}_{2} \mathrm{O}_{3}$ coating with a thickness of 200 $400 \mu \mathrm{m}$ was coated on the nickel and 5\%-aluminum interlayer using the same method.

To measure the insulating resistance between the $\mathrm{Al}_{2} \mathrm{O}_{3}$ coating and the SS316L substrate, silver conduc-

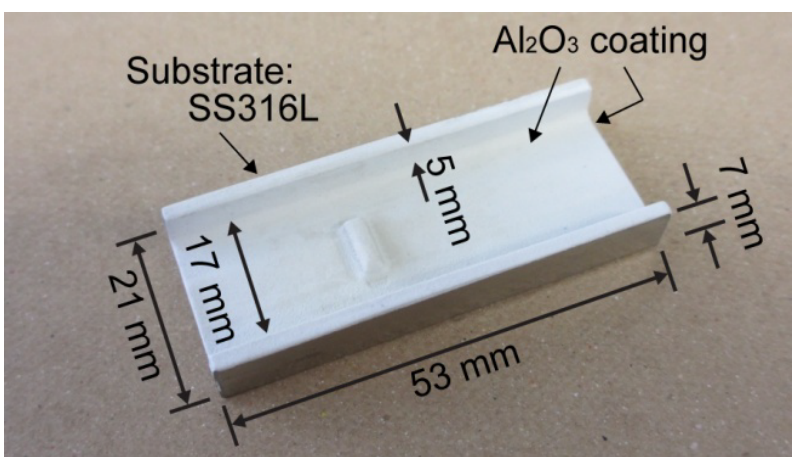

Fig. $9 \mathrm{Al}_{2} \mathrm{O}_{3}$-coated SS316L sample for the measurement of the insulating resistance before and after the heat-cycle tests.

tive paste was applied to the entire surface of the $\mathrm{Al}_{2} \mathrm{O}_{3}$ coating. The electrode made from SS316L with dimensions similar to those of the probe electrode was inserted in the samples. A DC voltage of $500 \mathrm{~V}$ was applied between the electrode and the SS316L substrate. Although the insulating resistance was measured under strict conditions compared with the actual conditions, this was sufficient to electrically insulate the probe electrode from the heat sink.

After the elimination of the paste, the heat-cycle test was performed in an argon gas atmosphere up to $200 \mathrm{cy}-$ cles. The samples were heated from the SS316L side. The temperature of the $\mathrm{Al}_{2} \mathrm{O}_{3}$ coating was measured with a thermocouple that was placed in contact with the coating surface and fixed with graphitic adhesives. The initial temperature was set to $50^{\circ} \mathrm{C}$, which corresponds to the temperature of the heat sink. The surface temperature of the aluminum coating was heated to $150^{\circ} \mathrm{C}$ with a similar rate of increase as that predicted by FEM analysis. The insulating resistance after the heat-cycle test indicated that no clear degradation of the insulating resistance was observed. Therefore, the $\mathrm{Al}_{2} \mathrm{O}_{3}$ coating was applied to the inner surface of the electrode holder and insulating plate of the Langmuir probe to insulate the probe electrode from the heat sink.

\section{Summary}

Four sets of 44-channel Langmuir probes have been developed and manufactured for the JT-60SA lower divertor. To minimize the reduction of the heat removal performance of the divertor, the Langmuir probes are installed in the lateral side of the water-cooled copper alloy heat sinks without embedding them in the divertor tiles. The heat sinks with the Langmuir probes are installed on the toroidal edge of the divertor cassettes, which corresponds to the upstream side of the SOL plasma flow. The Langmuir probes can be replaced by removing the divertor cassettes from the vacuum vessel. The Langmuir probes are installed at four toroidal positions (Sections P-3, 4, 9, and 15). Al- 
though the distance between the adjacent electrode heads of the Langmuir probes installed on the divertor targets is $27 \mathrm{~mm}$, shifting in the poloidal direction of the Langmuir probes installed on the Section P-3 and 9 divertor targets provides the minimum spatial resolution of $13.5 \mathrm{~mm}$. The spatial resolution of the other region is determined based on the decay length of the electron density.

To install the Langmuir probes on the toroidal edge of the divertor cassettes with a gap width of $10 \mathrm{~mm}$, the toroidal thickness of the Langmuir probe is limited to $8 \mathrm{~mm}$. This is achieved by applying an $\mathrm{Al}_{2} \mathrm{O}_{3}$ coating on the inner surface of the probe holder and the insulating plate made of SS316L to insulate the probe electrodes from the heat sinks. This provides the structure with the advantage of mechanical strength. Brazing of the connectors to the probe electrodes also reduces the toroidal thickness of the Langmuir probes. Before applying the coating and brazing technologies to the Langmuir probes, it is confirmed that the adhesion and conduction between the nickel and the CFCs and the insulating resistance of the $\mathrm{Al}_{2} \mathrm{O}_{3}$ coating are not clearly deteriorated by the heat cycles. Evaluation of the ion saturation current indicates that the Langmuir probes for the divertor targets can measure the plasma parameters expected at the divertor targets in JT-60SA. Expansion of the base of the probe electrode provides a decrease of the rooftop temperature to less than $1000^{\circ} \mathrm{C}$ for heat flux values of $1 \mathrm{MW} / \mathrm{m}^{2}$ for $100 \mathrm{~s}$ and $10 \mathrm{MW} / \mathrm{m}^{2}$ for $5 \mathrm{~s}$. This temperature is sufficiently low to avoid thermionic electron emission. In addition, the temperature of the probe electrode decreases to the initial temperature $\left(200^{\circ} \mathrm{C}\right)$ before the next plasma discharge, indicating that the time intervals between discharges are not limited by the temperature of the probe electrode.
The Langmuir probes enable us to measure the divertor plasma under the high heat load on the divertor. These Langmuir probes will also be applied to the other fusion experimental devices that include a cassette type of divertor such as ITER.

\section{Acknowledgments}

The authors thank Okazaki Manufacturing Company for manufacturing the Langmuir probes, and thank Dr. J.G. Watkins of Sandia National Laboratories, USA for information on the divertor probe design in DIII-D.

[1] N. Asakura et al., J. Nucl. Mater. 363-365, 41 (2007).

[2] C.S. Pitchery and P.C. Stangebyz, Plasma Phys. Control. Fusion 39, 779 (1997).

[3] A. Loarte et al., Nucl. Fusion 47, S128 (2007).

[4] P.C. Stangeby, The Plasma Boundary of Magnetic Fusion Devices (Taylor \& Francis, New York, 2000) p.477.

[5] S. Ishida et al., Nucl. Fusion 51, 094018 (2011).

[6] Y. Kamada et al., Nucl. Fusion 51, 073011 (2011).

[7] G.F. Matthews et al., Control. Plasma Phys. 36, 29 (1996).

[8] J.G. Watkins et al., Rev. Sci. Instrum. 79, 10F125 (2008).

[9] S. Sakurai et al., Fusion Eng. Des. 82, 1767 (2007).

[10] P.C. Stangeby, The Plasma Boundary of Magnetic Fusion Devices (Taylor \& Francis, New York, 2000) p.92.

[11] P.C. Stangeby, The Plasma Boundary of Magnetic Fusion Devices (Taylor \& Francis, New York, 2000) p.79.

[12] W. Fundamenski et al., Nucl. Fusion 51, 083028 (2011).

[13] K. Masaki et al., Fusion Eng. Des. 87, 742 (2012).

[14] K. Shimizu et al., Nucl. Fusion 49, 065028 (2009).

[15] H. Kawashima et al., J. Nucl. Mater. 415, S948 (2011).

[16] M. Wischmeier et al., J. Nucl. Mater. 390-391, 250 (2009).

[17] N. Asakura et al., J. Nucl. Mater. 290-293, 825 (2001).

[18] A. Loarte et al., Nucl. Fusion 38, 331 (1998).

[19] K. Hoshino et al., J. Plasma Fusion Res. SERIES 9, 592 (2010). 